\title{
ESTABLECIMIENTO Y MULTIPLICACIÓN IN VITRO DE PAPAYAS DE MONTANA: Vasconcellea chachapoyensis Y Vasconcellea x Heilbornii
}

\author{
Yoiner K. Lapiz-Culqui ${ }^{1}$, José J. Tejada-Alvarado ${ }^{1}$, Jegnes B. Meléndez-Mori ${ }^{1}$, \\ Nuri C. Vilca-Valqui ${ }^{1}$, Eyner Huaman-Huaman ${ }^{1}$ y Manuel Oliva ${ }^{1}$
}

\begin{abstract}
RESUMEN
La aplicación de las técnicas de cultivo de tejidos en especies de Vasconcellea es una alternativa viable para la propagación de estas especies. Sin embargo, la eficiencia de estas herramientas está condicionada por factores como la contaminación de explantes, el efecto de los reguladores de crecimiento y las especies que se desea propagar. El presente estudio tuvo como objetivo evaluar protocolos de desinfección superficial, con diferentes concentraciones de $\mathrm{NaClO}$ y $\mathrm{Ca}(\mathrm{ClO})_{2}$ y tiempos de

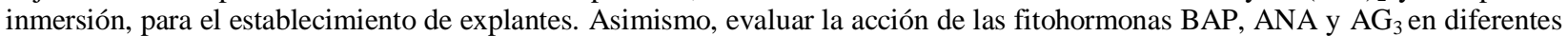
concentraciones en la multiplicación in vitro de segmentos nodales de Vasconcellea chachapoyensis y Vasconcellea x heilbornii. Los experimentos se condujeron bajo un diseño completamente al azar con arreglo factorial y los datos se analizaron con la prueba de Kruskal Wallis. Los resultados muestran que al utilizar $\mathrm{NaClO}$ al 1,5\% por 10 min se alcanzó solo $10 \%$ de contaminación, siendo $V$. chachapoyensis la especie que logró mayor viabilidad de explantes en la etapa de establecimiento. Por otro lado, el medio suplementado con BAP generó mayor número de brotes y hojas, mientras que el AG $_{3}$ indujo mayor elongación de los brotes. Además, cabe mencionar que la especie influyó en la multiplicación, siendo $V$. x heilbornii la de mejor desarrollo morfológico. Estos resultados señalan la eficacia e importancia de los desinfectantes y reguladores de crecimiento durante el cultivo de tejidos, y representan una alternativa propicia para la micropropagación de especies del género Vasconcellea.

Palabras-clave adicionales: Cultivo de tejidos, desinfección de explantes, reguladores de crecimiento vegetal, segmentos nodales
\end{abstract}

\begin{abstract}
In vitro establishment and multiplication of mountain papayas: Vasconcellea chachapoyensis and Vasconcellea $\mathbf{x}$ heilbornii The application of tissue culture techniques in Vasconcellea species is a viable alternative for the propagation of these species. However, the efficiency of these tools is conditioned by factors such as contamination of explants, the effect of growth regu lators and the species to be propagated. The present study aimed to evaluate sterilization protocols with different concentrations of $\mathrm{NaClO}$ and $\mathrm{Ca}(\mathrm{ClO})_{2}$ as well as different immersion times for the aseptic establishment of explants. Likewise, it was evaluated the action of the phytohormones BAP, ANA and $\mathrm{AG}_{3}$ in different concentrations in the in vitro multiplication of nodal segments of Vasconcellea chachapoyensis and Vasconcellea $\mathrm{x}$ heilbornii. The experiments were conducted under a completely randomized design with factorial arrangement, and the data were analyzed with the Kruskal Wallis test. The results showed that when using $1.5 \% \mathrm{NaClO}$ for $10 \mathrm{~min}$, only $10 \%$ contamination occurred, with $V$. chachapoyensis being the species that achieves the highest viability of explants in the establishment stage. On the other hand, the medium supplemented with BAP generated a greater number of shoots and leaves, while $\mathrm{AG}_{3}$ induced greater shoot elongation. In addition, it is worth mentioning that the species influenced multiplication, with $V$. x heilbornii being the species with the best morphological development. These results indicate the efficacy and importance of disinfectants and growth regulators during tissue culture, being a favorable alternative for the micropropagation of species of the genus Vasconcellea.
\end{abstract}

Additional keywords: Explant disinfection, nodal segments, plant growth regulators, tissue culture

INTRODUCCIÓN

El género Vasconcellea (Caricaceae) reúne a las especies conocidas como papayas de montaña, procedentes del centro y sur de América (Scheldeman et al., 2007; Robles et al., 2016).

Recibido: Diciembre 15, 2020

Aceptado: Marzo 20, 2021

${ }^{1}$ Instituto de Investigación para el Desarrollo Sustentable de Ceja de Selva (INDES-CES), Universidad Nacional Toribio Rodríguez de Mendoza (UNTRM), Chachapoyas 01001, Perú. e-mail: kalinlapiz@gmail.com, tejada.01634@gmail.com, jbenjamin@indes-ces.edu.pe (autor de correspondencia), nuricarito.02@gmail.com, eyner.huaman@untrm.edu.pe, soliva@indes-ces.edu.pe 
Muchos de sus frutos son comestibles, y en los últimos años han venido tomando mayor importancia gracias a su amplia utilidad alimenticia y potencial aprovechamiento de sus propiedades químicas (Posada, 2005; Scheldeman et al., 2011). Además, los frutos de Vasconcelleae son una rica fuente de látex con actividad proteolítica y lipolítica (Dhuique et al., 2001). Debido a su gran diversidad, es posible encontrar especies portadoras de genes de resistencia a enfermedades, tolerantes a bajas temperaturas y poseedores de atributos organolépticos especiales (Manshardt y Wenslaff, 1989; Drew et al., 1998; Scheldeman et al., 2007).

Dentro de esa amplia diversidad, destaca Vasconcellea $\mathrm{x}$ heilbornii, cuyos frutos son apreciados en los mercados internacionales debido a su sabor y aroma únicos, además de la ausencia de semillas (Cornejo et al., 2020). Debido a esta característica, su propagación es realizada mediante estacas, el cual es un método poco confiable debido al riesgo de transmisión de enfermedades (Posada, 2005; Jádan et al., 2016a; Cornejo et al., 2020).

Por otra parte, Vasconcellea chachapoyensis posee frutos que se utilizan de forma artesanal para elaboración de dulces, mermeladas y yogurt. $\mathrm{Su}$ reciente descripción como nueva especie (Tineo et al., 2020) abre una brecha para el estudio de sus propiedades y potencial agronómico, así como para iniciar a explorar las estrategias para su propagación.

Como respuesta a la necesidad de obtener plantas sanas, de calidad y uniformidad genética de estas especies, es ineludible el aporte de la propagación in vitro. Los estudios actuales buscan nuevas alternativas en los protocolos de micropropagación de $V$. x heilbornii, pues pese a que diversos estudios informan sobre el uso de fitorreguladores para su multiplicación, una de las limitantes es el bajo éxito logrado durante el establecimiento in vitro (Jádan et al., 2016a; Millones, 2019), mientras que para $V$. chachapoyensis probablemente no existan estudios relacionados. Lograr el cultivo in vitro de tejidos plantea retos, como controlar la contaminación microbiana que afecta el desarrollo del explante (Hernández y González 2010; Jádan et al., 2016b). Por otro lado, el uso de reguladores de crecimiento ha mostrado efectos muy variables en la micropropagación (Campos et al., 2020), por lo que es necesario generar nuevos conocimientos para la propagación de estas especies.

En ese contexto, el objetivo del presente estudio fue evaluar protocolos de desinfección superficial con diferentes concentraciones de $\mathrm{NaClO}$ y $\mathrm{Ca}(\mathrm{ClO})_{2}$ y tiempos de inmersión para el establecimiento de explantes. Asimismo, evaluar la acción de las fitohormonas BAP, ANA y $\mathrm{AG}_{3}$ en diferentes concentraciones en la multiplicación in vitro de segmentos nodales de $V$. chachapoyensis y $V$. x heilbornii.

\section{MATERIALES Y MÉTODOS}

Material vegetal. El germoplasma se recolectó de huertos ubicados en la región Amazonas, en el norte de Perú. Se colectaron estacas de $V$. chachapoyensis (distrito de Olleros; $6^{\circ} 02^{\prime} \mathrm{S}$ y $77^{\circ}$ 38' W; $3041 \mathrm{msnm}$ ) y $V$. x heilbornii (distrito de Lamud; 6 $6^{\circ} 08^{\prime} \mathrm{S}$ y $77^{\circ} 57^{\prime} \mathrm{W}$; $2311 \mathrm{msnm}$ ) y se cultivaron en vivero durante 6 meses a una temperatura media de $20 \pm 2{ }^{\circ} \mathrm{C}$ y una humedad relativa mayor a $70 \%$. Durante ese periodo recibieron tratamiento sanitario $\left(2 \mathrm{~mL} \cdot \mathrm{L}^{-1}\right.$ de sulfato de cobre pentahidratado $+1 \mathrm{~mL} \cdot \mathrm{L}^{-1}$ cipermetrina, cada 15 días) y estimulación hormonal $\left(2 \mathrm{~mL} \cdot \mathrm{L}^{-1}\right.$ de Gib-bex ${ }^{\circledR}$, cada 20 días) para asegurar el desarrollo de brotes sanos y vigorosos.

Extracción de explantes. Se extrajeron segmentos nodales $(4 \mathrm{~cm})$ con una o dos yemas axilares y se introdujeron en una solución de 150 $\mathrm{mg} \cdot \mathrm{L}^{-1}$ de ácido ascórbico (solución antioxidante). A continuación, se sometieron a un proceso de limpieza superficial mediante inmersión en solución jabonosa por 15 minutos y solución de fungicida (Benomyl $1 \mathrm{~g} \cdot \mathrm{L}^{-1}$ ) por 30 minutos. Al finalizar cada limpieza se realizó un triple enjuague con agua estéril. Finalmente, los segmentos nodales se cortaron en fragmentos de 1,5 a $2 \mathrm{~cm}$ de longitud.

Fase de establecimiento in vitro. En área aséptica, los explantes se sumergieron en alcohol $(70 \%$ v/v) durante 30 segundos y luego se enjuagaron con agua destilada estéril. Para completar la desinfección superficial se evaluó el efecto de $\mathrm{Ca}(\mathrm{ClO})_{2}$ y $\mathrm{NaClO}$ (agentes desinfectantes), en distintas concentraciones $\mathrm{y}$ tiempos de inmersión (Cuadro 1).

Los explantes se establecieron en medio de cultivo MS (Murashigue y Skoog, 1962), 
suplementado con sacarosa (3\%), el producto PPM (Plant Preservation Mixture) $\left(1 \mathrm{~mL} \cdot \mathrm{L}^{-1}\right)$ y sin reguladores de crecimiento. El pH se ajustó a 5,8 , se agregó agar (6\%) y se esterilizó en autoclave a $120{ }^{\circ} \mathrm{C}$ y presión de $\sim 1 \mathrm{~kg} \cdot \mathrm{cm}^{-2}$ durante $20 \mathrm{~min}$. De cada especie se sembraron individualmente 120 explantes en tubos de ensayo que contenían $15 \mathrm{~mL}$ de medio de cultivo y se incubaron en sala de crecimiento a $24 \pm 2{ }^{\circ} \mathrm{C}, 16 \mathrm{~h}$ de fotoperiodo y 3000 lux de intensidad de luz suministrada por lámparas fluorescentes blancas. Después de 21 días se evaluó el porcentaje de explantes oxidados, contaminados y viables.

Cuadro 1. Tratamientos de desinfección evaluados en el establecimiento in vitro de explantes de Vasconcellea

\begin{tabular}{ccc}
\hline Desinfectante & $\begin{array}{c}\text { Concentración } \\
(\%)\end{array}$ & $\begin{array}{c}\text { Tiempo } \\
(\text { min })\end{array}$ \\
\hline & 1,0 & 5 \\
& 1,5 & 5 \\
$\mathrm{Ca}(\mathrm{ClO})_{2}$ & 2,0 & 5 \\
& 1,0 & 10 \\
& 1,5 & 10 \\
& 2,0 & 10 \\
\hline \multirow{2}{*}{$\mathrm{NaClO}$} & 1,0 & 5 \\
& 1,5 & 5 \\
& 2,0 & 5 \\
& 1,0 & 10 \\
& 1,5 & 10 \\
& 2,0 & 10 \\
\hline
\end{tabular}

Fase de multiplicación in vitro. Para esta fase se utilizaron explantes procedentes del establecimiento in vitro bajo tratamiento con $1,5 \%$ de $\mathrm{NaClO}$ durante 10 minutos. Estos fueron subcultivados en medio MS, suplementado con 0,2 y $0,5 \mathrm{mg} \cdot \mathrm{L}^{-1}$ de $\mathrm{AG}_{3}$, ANA o BAP, según fuese el tratamiento (Cuadro 2). Los cultivos se incubaron en la sala de crecimiento con las condiciones antes señaladas. Luego de 45 días de cultivo se evaluó el número de brotes, longitud de brotes y número de hojas.

Diseño experimental y análisis de datos. El experimento se condujo bajo un diseño completamente al azar con arreglo factorial $2 \times 3 \times 2$, con 10 repeticiones por tratamiento. Los datos se sometieron a la prueba de normalidad de
Shapiro-Wilk y homogeneidad de varianzas (estadístico de Levene), y dado que no se ajustaron a una distribución normal se aplicó la prueba no paramétrica de Kruskal-Wallis. El análisis se realizó utilizando el programa InfoStat versión 2017.

Cuadro 2. Tratamientos evaluados en la multiplicación in vitro de $V$. chachapoyensis y V. x heilbornii.

\begin{tabular}{|c|c|c|}
\hline Especie & $\begin{array}{l}\text { Reguladores } \\
\text { de crecimiento }\end{array}$ & $\begin{array}{l}\text { Concentración } \\
\left(\mathrm{mg} \cdot \mathrm{L}^{-1}\right)\end{array}$ \\
\hline \multirow{6}{*}{ V. chachapoyensis } & $\mathrm{AG}_{3}$ & 0,2 \\
\hline & ANA & 0,2 \\
\hline & BAP & 0,2 \\
\hline & $\mathrm{AG}_{3}$ & 0,5 \\
\hline & ANA & 0,5 \\
\hline & BAP & 0,5 \\
\hline \multirow{6}{*}{$V . \mathrm{x}$ heilbornii } & $\mathrm{AG}_{3}$ & 0,2 \\
\hline & ANA & 0,2 \\
\hline & BAP & 0,2 \\
\hline & $\mathrm{AG}_{3}$ & 0,5 \\
\hline & ANA & 0,5 \\
\hline & BAP & 0,5 \\
\hline
\end{tabular}

\section{RESULTADOS}

Establecimiento in vitro. $\mathrm{El}$ análisis muestra diferencias significativas $(P \leq 0,05)$ en el establecimiento in vitro de Vasconcellea (Cuadro 3). El nivel de oxidación de explantes varió entre $10 \mathrm{y}$ $70 \%$. Los explantes de $V$. chachapoyensis y $V . \mathrm{x}$ heilbornii tratados con $\mathrm{NaClO}$ al $1 \%$ durante 5 minutos registraron la oxidación más baja. Asimismo, se observó que independientemente del tipo de desinfectante, el uso de una concentración del $2 \%$ hizo que la oxidación superara el $40 \%$.

Respecto a los niveles de contaminación, se observó que bajo la desinfección con $\mathrm{NaClO}$ al $1,5 \%$ por $10 \mathrm{~min}$, solo $10 \%$ de los explantes de las dos especies presentaron signos de contaminación (hongos y bacterias). Pero cuando se utilizó la concentración de desinfectante al $1 \%$, ya fuese con $\mathrm{Ca}(\mathrm{ClO})_{2}$ o $\mathrm{NaClO}$, y un tiempo de inmersión de $5 \mathrm{~min}$, el porcentaje de explantes contaminados fue superior a $60 \%$ (Cuadro 3). La viabilidad de los explantes fue inversamente proporcional a su nivel de contaminación. 
Cuadro 3. Efecto de diferentes desinfectantes, concentraciones y tiempos de inmersión en el establecimiento in vitro de $V$. chachapoyensis y $V$. x heilbornii

\begin{tabular}{|c|c|c|c|c|c|c|}
\hline \multirow{3}{*}{ Tratamientos } & \multicolumn{3}{|c|}{ Vasconcellea chachapoyensis } & \multicolumn{3}{|c|}{ Vasconcellea $\mathrm{x}$ heilbornii } \\
\hline & Oxidación & Contaminación & Viabilidad & Oxidación & Contaminación & Viabilidad \\
\hline & \multicolumn{3}{|c|}{$\%$} & \multicolumn{3}{|c|}{$\%$} \\
\hline $\mathrm{Ca}(\mathrm{ClO})_{2} \times 1 \times 5$ & $20,0 \mathrm{de}$ & $70,0 \mathrm{ab}$ & $10,0 \mathrm{~d}$ & $20,0 \mathrm{~cd}$ & $60,0 \mathrm{ab}$ & $20,0 \mathrm{~b}$ \\
\hline $\mathrm{Ca}(\mathrm{ClO})_{2} \times 1 \times 10$ & $40,0 \mathrm{bc}$ & $40,0 \mathrm{bc}$ & $20,0 \mathrm{c}$ & $40,0 \mathrm{ab}$ & $50,0 \mathrm{bc}$ & $10,0 \mathrm{c}$ \\
\hline $\mathrm{Ca}(\mathrm{ClO})_{2} \times 1,5 \times 5$ & $30,0 \mathrm{~cd}$ & $30,0 \mathrm{~cd}$ & $40,0 \mathrm{abc}$ & $30,0 \mathrm{bc}$ & $40,0 \mathrm{~cd}$ & $30,0 \mathrm{ab}$ \\
\hline $\mathrm{Ca}(\mathrm{ClO})_{2} \times 1,5 \times 10$ & $40,0 \mathrm{bc}$ & $10,0 \mathrm{e}$ & $50,0 \mathrm{ab}$ & $20,0 \mathrm{~cd}$ & 20,0 ef & $60,0 \mathrm{a}$ \\
\hline $\mathrm{Ca}(\mathrm{ClO})_{2} \times 2 \times 5$ & $40,0 \mathrm{bc}$ & $30,0 \mathrm{~cd}$ & $30,0 \mathrm{bc}$ & $40,0 \mathrm{ab}$ & $40,0 \mathrm{~cd}$ & $20,0 \mathrm{~b}$ \\
\hline $\mathrm{Ca}(\mathrm{ClO})_{2} \times 2 \times 10$ & $60,0 \mathrm{ab}$ & $30,0 \mathrm{~cd}$ & $10,0 \mathrm{~d}$ & $70,0 \mathrm{a}$ & 20,0 ef & $10,0 \mathrm{c}$ \\
\hline $\mathrm{NaClO} \times 1 \times 5$ & $10,0 \mathrm{e}$ & $80,0 \mathrm{a}$ & $10,0 \mathrm{~d}$ & $10,0 \mathrm{~d}$ & 80,0 a & $10,0 \mathrm{c}$ \\
\hline $\mathrm{NaClO} \times 1 \times 10$ & 20,0 de & $70,0 \mathrm{ab}$ & $10,0 \mathrm{~d}$ & $20,0 \mathrm{~cd}$ & $70,0 \mathrm{ab}$ & $10,0 \mathrm{c}$ \\
\hline $\mathrm{NaClO} \times 1,5 \times 5$ & $10,0 \mathrm{e}$ & $60,0 \mathrm{ab}$ & $30,0 \mathrm{bc}$ & $20,0 \mathrm{~cd}$ & $50,0 \mathrm{bc}$ & $30,0 \mathrm{ab}$ \\
\hline $\mathrm{NaClO} \times 1,5 \times 10$ & 20,0 de & $10,0 \mathrm{e}$ & $70,0 \mathrm{a}$ & $30,0 \mathrm{bc}$ & $10,0 \mathrm{f}$ & $60,0 \mathrm{a}$ \\
\hline $\mathrm{NaClO} \times 2 \times 5$ & $50,0 \mathrm{ab}$ & $40,0 \mathrm{bc}$ & $10,0 \mathrm{~d}$ & 50,0 a & $30,0 \mathrm{de}$ & $20,0 \mathrm{~b}$ \\
\hline $\mathrm{NaClO} \times 2 \times 10$ & $70,0 \mathrm{a}$ & $20,0 \mathrm{de}$ & $10,0 \mathrm{~d}$ & $70,0 \mathrm{a}$ & 20,0 ef & $10,0 \mathrm{c}$ \\
\hline
\end{tabular}

Promedios con letras distintas entre filas de cada columna indican diferencias significativas (Kruskal-Wallis, $P \leq 0,05$ )

Multiplicación in vitro. El Cuadro 4 muestra diferencias significativas $(P \leq 0,05)$ en las variables morfométricas. Se observa que el mayor número de brotes por explante se registró en la combinación de $V$. x heilbornii y BAP (independientemente de la concentración), con un promedio superior a 3,90 brotes, mientras que el menor número de brotes $(1,40)$ se registró en los explantes (de ambas especies) tratados con 0,5 $\mathrm{mg} \cdot \mathrm{L}^{-1}$ de $\mathrm{AG}_{3}$.

El número promedio de hojas varió entre 1,70 y 4,30 por explante, con un máximo registrado en $V$. x heilbornii y el mínimo en $V$. chachapoyensis; ambos valores fueron alcanzados bajo el tratamiento de $0,5 \mathrm{mg} \cdot \mathrm{L}^{-1}$ de BAP. En cuanto a la longitud de los nuevos brotes, se observó que los explantes de $V$. x heilbornii cultivados en medio suplementado con $0,5 \mathrm{mg} \cdot \mathrm{L}^{-1}$ de BAP presentaron brotes con $2,71 \mathrm{~cm}$ de longitud, mientras que los brotes de $V$. chachapoyensis alcanzaron una longitud de $2,86 \mathrm{~cm}$ cuando se cultivaron con 0,5 $\mathrm{mg} \mathrm{L}^{-1}$ de $\mathrm{AG}_{3}$, siendo estos resultados superiores al efecto inducido por la aplicación de la fitohormona ANA.

\section{DISCUSIÓN}

Establecimiento in vitro. La obtención de explantes exentos de contaminantes es uno de los puntos críticos para el desarrollo de protocolos eficientes de micropropagación, debido a la presencia de agentes patógenos que contaminan los tejidos (Posada, 2005), y que pueden producir cuantiosas pérdidas de material (Hernández y González, 2010; Gerónimo et al., 2016).

En el presente estudio, el tratamiento de desinfección más eficiente fue $\mathrm{NaClO}$ al $1,5 \%$ por 10 minutos, pues solo el $10 \%$ de explantes manifestó contaminación. Bajo el mismo protocolo de desinfección, Jádan et al. (2016b) reportaron que solo se redujo en un $55 \%$ la contaminación por bacterias y $3 \%$ por hongos. Por su parte, Solis et al. (2012) indicaron que el uso de alcohol (70 \%) por 30 segundos y $\mathrm{NaClO}$ (1\%) durante 10 minutos tuvo alta eficiencia en la desinfección de explantes provenientes de plantas de invernadero.

La diferencia entre los estudios anteriores puede estar relacionada con la manipulación del material vegetal, porque ésta es una de las fuentes más comunes de contaminación (Ticona y Triguero, 2020). Además, se confirma que la desinfección superficial del explante está condicionada por factores como el tipo, concentración y tiempo de exposición a los agentes desinfectantes, pero también por la procedencia del explante (Posada et al., 2004; Ticona y Triguero, 2019). Establecer protocolos de desinfección superficial eficientes tiene un rol fundamental porque puede determinar el éxito o 
fracaso del cultivo de tejidos (Okereke et al., 2016) por lo que, además de los factores antes señalados, es importante la limpieza inicial del explante debido a que puede disminuir la cantidad de microorganismos en la superficie, permitiendo que el desinfectante actúe con mayor eficiencia.

Cuadro 4. Efecto de diferentes reguladores de crecimiento y concentraciones en la multiplicación in vitro de $V$. chachapoyensis y $V$. x heilbornii

\begin{tabular}{|c|c|c|c|}
\hline Tratamientos & Número de brotes & Número de hojas & Longitud de brote $(\mathrm{cm})$ \\
\hline V. chachapoyensis $\times \mathrm{AG}_{3} \times 0,2$ & $1,60 \mathrm{bc}$ & $1,80 \mathrm{c}$ & $2,34 \mathrm{ab}$ \\
\hline V. chachapoyensis $\times \mathrm{AG}_{3} \times 0,5$ & $1,40 \mathrm{c}$ & $2,20 \mathrm{c}$ & $2,86 \mathrm{a}$ \\
\hline$V$. chachapoyensis $\mathrm{x}$ ANA $\mathrm{x} 0,2$ & $1,50 \mathrm{bc}$ & $1,90 \mathrm{c}$ & $0,79 \mathrm{e}$ \\
\hline V. chachapoyensis $\mathrm{x}$ ANA $\times 0,5$ & $1,70 \mathrm{bc}$ & $2,20 \mathrm{c}$ & 1,05 ef \\
\hline V. chachapoyensis $\mathrm{x}$ BAP $\mathrm{x} 0,2$ & $1,90 \mathrm{bc}$ & $1,80 \mathrm{c}$ & $1,18 \mathrm{def}$ \\
\hline$V$. chachapoyensis $\mathrm{x}$ BAP $\mathrm{x} 0,5$ & $2,20 \mathrm{~b}$ & $1,70 \mathrm{c}$ & $1,86 \mathrm{bc}$ \\
\hline$V . \times$ heilbornii $\times \mathrm{AG}_{3} \times 0,2$ & $1,60 \mathrm{bc}$ & $1,80 \mathrm{c}$ & 1,53 cde \\
\hline V. $\times$ heilbornii $\times \mathrm{AG}_{3} \times 0,5$ & $1,40 \mathrm{c}$ & $1,80 \mathrm{c}$ & $2,18 \mathrm{abc}$ \\
\hline V. $\mathrm{x}$ heilbornii $\mathrm{x}$ ANA $\mathrm{x} 0,2$ & $1,60 \mathrm{bc}$ & $1,90 \mathrm{c}$ & $1,02 \mathrm{e}$ \\
\hline V. $\mathrm{x}$ heilbornii $\mathrm{x}$ ANA $\mathrm{x} 0,5$ & $2,00 \mathrm{bc}$ & $2,40 \mathrm{bc}$ & $1,17 \mathrm{def}$ \\
\hline V. $\mathrm{x}$ heilbornii $\mathrm{x}$ BAP $\times 10,2$ & $3,90 \mathrm{a}$ & $3,20 \mathrm{ab}$ & $1,64 \mathrm{bcd}$ \\
\hline$V . \times$ heilbornii $\times$ BAP $\times 0,5$ & $4,60 \mathrm{a}$ & $4,30 \mathrm{a}$ & $2,71 \mathrm{a}$ \\
\hline
\end{tabular}

Promedios con letras distintas entre filas de cada columna indican diferencias significativas (Kruskal-Wallis, $P \leq 0,05$ )

Otro de los factores a considerar durante el establecimiento in vitro es la oxidación de explantes. El proceso oxidativo es frecuente durante esta fase, pues mucho de los explantes liberan exudados que producen el oscurecimiento del medio y el explante, con subsecuente muerte del tejido vegetal (Azofeifa, 2009). Una de las causas señaladas es el uso de altas concentraciones de desinfectantes, los cuales pueden causar efectos tóxicos, sobre todo en explantes jóvenes (Agramonte et al., 2001). Los resultados de este estudio confirman que el incremento en los niveles de oxidación del explante está relacionado con el aumento en la concentración y tiempo de exposición de éste al desinfectante. Por otro lado, se podría sugerir que las diferentes respuestas de los explantes se pueden relacionar también con el nivel de tolerancia propia de cada especie.

En general, a los 21 días de establecimiento, los explantes de ambas especies de Vasconcellea presentaron resultados alentadores, aunque $V$. chachapoyensis registró mejor viabilidad (70 \%). Se observaron explantes con formación de un brote $(<1 \mathrm{~cm})$, aunque en esta etapa no se emplearon fitorreguladores. Según Millones
(2019), el uso de reguladores como ANA (0,25 $\left.\mathrm{mg} \mathrm{L}{ }^{-1}\right)$, AIA $\left(0,5 \mathrm{mg} \cdot \mathrm{L}^{-1}\right)$ y BAP $\left(1 \mathrm{mg} \cdot \mathrm{L}^{-1}\right)$ no mejora la respuesta morfométrica en yemas axilares de $V$. x heilbornii. Por lo tanto, como objetivo inicial es importante obtener vitroplantas libres de contaminantes, de modo que se garantice la mayor disponibilidad de material para la siguiente fase del cultivo de tejidos.

Multiplicación in vitro. En la fase de multiplicación se registró el desarrollo de más de 4 hojas y brotes en explantes de $V$. x heilbornii cultivados en $0,5 \mathrm{mg} \cdot \mathrm{L}^{-1}$ de BAP; por el contrario, bajo esta concentración de citoquinina se redujo considerablemente la formación de estructuras vegetativas en $V$. chachapoyensis. Varios factores pueden influir en la regeneración de plantas mediante cultivo in vitro, entre ellas el genotipo (Chong et al., 2018). Por ejemplo, Vélez et al. (2015) informaron que segmentos nodales de $V$. stipulata (uno de los progenitores de $V$. X heilbornii) cultivados en medio suplementado con ANA y $\operatorname{BAP}(0,54 \quad$ y $2 \quad \mu M)$ indujeron la formación de hasta 4 nuevos brotes por explante. Esta capacidad de regeneración puede sugerir que la respuesta superior mostrada por $V$. x heilbornii está asociada a un rasgo hereditario de uno de sus 
progenitores. Las variaciones registradas también pueden deberse a que el requerimiento de reguladores de crecimiento en el cultivo in vitro es extremadamente variable y que las respuestas a la inducción de brotes dependen del contenido endógeno de cada especie (Campos et al., 2020). Los resultados corroboran el efecto de BAP como estimulador de la división de tejidos no meristemáticos, siendo altamente efectivo para estimular la formación de brotes (Solis et al., 2012; Gil et al., 2016).

Por su parte, el uso del $\mathrm{AG}_{3}$ se mostró bastante efectivo en la elongación de los brotes, demostrando que los fitorreguladores tienen funciones diferentes y complementarias. De acuerdo con Uribe et al. (2008), el crecimiento del tallo es, probablemente, el efecto fisiológico más importante inducido por el uso de $\mathrm{AG}_{3}$. Sin embargo, se ha reportado que las concentraciones óptimas para la micropropagación se encuentran hasta en $1 \mathrm{mg} \cdot \mathrm{L}^{-1}$, debido a que concentraciones superiores pueden derivar en efectos tóxicos (Ramesh et al. 2018; Palei et al., 2019). Los niveles que se consideren adecuados pueden variar con cada especie, pero también con el efecto sinérgico entre reguladores de crecimiento (Gil et al., 2016). Es preciso mencionar que, si bien hubo éxito en estas primeras etapas del cultivo de tejidos, falta aún la parte de enraizamiento, ya que para considerar la factibilidad del cultivo in vitro en estos genotipos lo ideal sería lograr un medio de establecimiento o de multiplicación en el que además del establecimiento per se, haya también formación, proliferación y elongación de raíces.

\section{CONCLUSIONES}

Se evidencia que el $\mathrm{NaClO}$ presenta mejores efectos en la desinfección superficial de explantes de Vasconcellea y que tanto la concentración como el tiempo de inmersión en el desinfectante influyen en la eliminación de los agentes contaminantes.

Los reguladores de crecimiento mostraron efectos significativos en la fase de multiplicación, siendo BAP $\left(0,5 \mathrm{mg} \cdot \mathrm{L}^{-1}\right)$ el regulador de crecimiento más adecuado para inducir la formación de estructuras vegetativas en explantes de $V$. x heilbornii y $V$. chachapoyensis. Asimismo, se comprobó que $\mathrm{AG}_{3}$ tiene activa participación en la elongación de brotes.

\section{AGRADECIMIENTOS}

Los autores agradecen el financiamiento realizado a través del proyecto SNIP $\mathrm{N}^{\circ} 312252$ "Creación del Servicio de un Laboratorio de Fisiología y Biotecnología Vegetal de la Universidad Nacional Toribio Rodríguez de Mendoza de Amazonas", ejecutado por el Instituto de Investigación para el Desarrollo Sustentable de Ceja de Selva.

\section{LITERATURA CITADA}

1. Agramonte-Peñalver, D., L. DelgadoFernández, A. Trocones-Boggiano, M. PérezPeralta, D. Ramírez-Aguilar y O. GutiérrezMartínez. 2001. Micropropagación del Eucalyptus grandis (Hill ex Maiden) a partir segmentos nodales. Biotecnología Vegetal 1(2): 109-114.

2. Azofeifa-Delgado, A. 2009. Problemas de oxidación y oscurecimiento de explantes cultivados in vitro. Agronomía Mesoamericana 20(1): 153-175.

3. Campos, J., M. Arteaga, S. Campos y J. Chico. 2020. Establecimiento de un protocolo de desinfección y micropropagación in vitro de "caoba" Swietenia macrophylla King (Meliaceae). Arnaldoa 27(1): 141-156.

4. Chong-Pérez, B., B. Carrasco, H. Silva, F. Herrera, K. Quiroz y R. García-Gonzales. 2018. Regeneration of highland papaya (Vasconcellea pubescens) from anther culture. Applications in Plant Sciences 6(9): e01182.

5. Cornejo-Franco, J.F., A. Medina-Salguero, F. Flores, E. Chica, S. Grinstead, D. Mollov y D.F. Quito-Avila. 2020. Exploring the virome of Vasconcellea x heilbornii: The first step towards a sustainable production program for babaco in Ecuador. European Journal of Plant Pathology 157: 961-968.

6. Dhuique-Mayer, C., Y. Caro, M. Pina, J. Ruales, M. Dornier y J. Graille. 2001. Biocatalytic properties of lipase in crude latex from babaco fruit (Carica pentagona). Biotechnology Letters 23(13): 1021-1024.

7. Drew, R.A., C.M. O’Brien y P.M. Magdalita. 1998. Development of Carica interspecific hybrids. Acta Horticulturae 461: 285-291. 
8. Gerónimo G.F., B.L.F. Pérez, G. Plata y V.J.G. Aguirre. 2016. Contaminantes en cultivo in vitro. In: Aguirre, G., J. Pierre y L. Leigue (eds.). Aplicación del Cultivo de Tejidos en la Multiplicación y Conservación de los Recursos Fitogenéticos. Universidad Mayor de San Simón. Cochabamba, Bolivia. pp. 57-68.

9. Gil-Rivero, A.E., E. López-Medina y A. López-Zabaleta. 2016. Efecto sinérgico del ácido indolacético, ácido giberélico y 6bencilaminopurina en la propagación in vitro de "papaya" Carica papaya L. (Caricaceae). Arnaldoa 23(2): 577-586.

10.Hernández, Y. y M. E. González. 2010. Efectos de la contaminación microbiana y oxidación fenólica en el establecimiento in vitro de frutales perennes. Cultivos Tropicales 31(4): 1-5.

11.Jádan, M.G., R. Gómez-Kosky y I. BermúdezCaraballoso. 2016a. Obtención de plantas madre de Vasconcellea x helbornii (Badillo) Badillo a partir de estacas en condiciones semicontroladas. Biotecnología vegetal 16(1): 13-20.

12.Jádan, M.G., K. Basantez, R. Gómez-Kosky y I. Bermúdez-Caraballoso. 2016b. Establecimiento in vitro de brotes de Vasconcellea $\mathrm{x}$ helbornii (Badillo) Badillo. Biotecnología Vegetal 16(2): 67-72.

13.Manshardt, R.M. y T.F. Wenslaff. 1989. Zygotic polyembryony in interspecific hybrids of Carica papaya and C. cauliflora. Journal of the American Society for Horticultural Science 114(4): 684-689.

14.Millones, C.E. 2019. Propagación in vitro de babaco (Vasconcellea $\mathrm{x}$ heilbornii) del distrito de Luya, región Amazonas. Revista Científica UNTRM: Ciencias Naturales e Ingeniería 2(1): 45-51.

15.Murashigue, T. y F. Skoog. 1962. A revised medium for rapid growth and bioassays with tobacco tissue cultures. Physiologia Plantarum 15(3): 473-497.

16.Okereke, O.E., H.O. Akanya, G.H. Ogbadu, E.C. Egwim, V.A. Etim y S.A. Akande. 2016. Development and optimization of a surface sterilization protocol for the tissue culture of Pleurotus tuber-regium (fr) sing. and Auricularia auricula-judae. International
Journal of Biochemistry, Bioinformatics and Biotechnology Studies 1(2): 1-9.

17.Palei, S., D.K. Dash y G.R. Rout. 2019. Standardization of in vitro protocol for plant regeneration of Carica papaya cv. Co8 through indirect organogénesis. Journal of Pharmacognosy and Phytochemistry 8(3): 1954-1956.

18.Posada, L., R. Gómez, J. Gallardo, M. Reyes y I. Herrera. 2004. Establecimiento in vitro de ápices de plantas de campo del hibrido cubano de papaya IBP 42-59. Biotecnología Vegetal 4(3): 153-158.

19.Posada, L. 2005. Aplicaciones de la biotecnología a la propagación de la papaya. Biotecnología Vegetal 5(2): 67-79.

20.Ramesh, A.N., D.B. Santhosh, D. Suresh, K. Radha, K. Rashmi y K. Hajira. 2018. In vitro regeneration of papaya (Carica papaya L.) Variety Surya. International Journal of Pure \& Applied Bioscience 6(4): 456-461.

21.Robles-Carrión, A.R., L. Herrera-Isla y R. Torres-Gutiérrez. 2016. El babaco (Vasconcellea heilbornii var. Pentagona Badillo). Principales agentes fitopatógenos y estrategias de control. Centro Agrícola 43(2): 83-92.

22.Scheldeman, X., L. Willemen, G. Coppens d'Eeckenbrugge, E. Romeijin-Peeters, M.T. Restrepo, J. Romero-Motoche et al. 2007. Distribution, diversity and environmental adaptation of highland papayas (Vasconcellea spp.) in tropical and subtropical America. Biodiversity and Conservation. 16(6): 18671884.

23.Scheldeman, X., T. Kyndt, G.C. Coppens d'Eeckenbrugge, R. Ming, R. Drew, B.V. Droogenbroeck et al. 2011. Vasconcellea. In: C. Kole (ed.). Wild Crop Relatives: Genomic and Breeding Resources. Tropical and Subtropical Fruits. Springer. Heidelberg, Germany. pp. 213-249.

24.Solis, R., J. Olivera y R.S. La Rosa. 2012. Propagación in vitro de Carica papaya var. PTM-331 a partir de meristemos apicales. Revista Peruana de Biología 18(3): 343-347.

25.Ticona, J. y M.L. Triguero. 2019. Evaluación de tres métodos de desinfección para el establecimiento in vitro de papaya (Carica 
papaya L.) en la Estación Experimental Sapecho. Revista de Investigación e Innovación Agropecuaria y de Recursos Naturales 6(1): 24-29.

26.Ticona, J. y M.L. Triguero. 2020. Evaluación del comportamiento in vitro de dos variedades de papaya (Carica papaya L.) mediante embriogénesis somática en la Estación Experimental Sapecho. Revista de Investigación e Innovación Agropecuaria y de Recursos Naturales 7(1): 55-61.

27.Tineo, D., D.E. Bustamante, M.S. Calderon, J.E. Mendoza, E. Huaman y M. Oliva. 2020. An integrative approach reveals five new species of highland papayas (Caricaceae, Vasconcellea) from northern Peru. PLoS ONE 15(12): e0242469.

28.Uribe, M.E., C. Delaveau, M. Garcés y R. Escobar. 2008. Efecto de asepsia y fitohormonas en el establecimiento in vitro de Berberidopsis corallina, a partir de segmentos nodales. Bosque 29(1): 58-64.

29.Vélez-Mora, D.P., R. Armijos y M. Jordán. 2015. Enhancement of germination, hyperhydricity control and in vitro shoot formation of Vasconcellea stipulata Badillo. Revista Colombiana de Biotecnología 18(2): 16-21. 